\title{
Nur verwirrt oder ernsthaft krank?
}

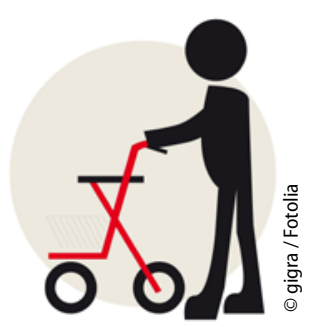

\author{
Ein Delir ist immer als akuter Notfall zu betrachten, der rasch abgeklärt werden muss. Wie das \\ diagnostische und therapeutische Vorgehen aussehen sollte, erläutert Prof. H.-J. Heppner im \\ folgenden Beitrag.
}

? Im Bereitschaftsdienst wurde ich zu einer 80-jährigen Patientin gerufen. Den Angehörigen war aufgefallen, dass sie „anders sei als sonst" und sehr unruhig war. Es bestand der Wunsch nach einem Beruhigungsmittel. Wie sollte man intervenieren?

I Die Patientin war im übertragenen Sinne „aus der Spur geraten“ (lat.: de lira = aus der Furche), litt also an einem Delir. Typisch ist der akute Beginn und der fluktuierende Verlauf. Es gibt drei Formen: hyperaktives (15\%), hypoaktives (25\%) und gemischtes Delir (50\%).

Das Delir ist immer nur Symptom einer akuten, therapiebedürftigen Erkrankung, wie Infektion, Sepsis, Harnverhalt, Trauma oder Folge eines operativen oder interventionellen Eingriffs. Auch Medikamente wie Analgetika (Opioide), Antibiotika (Gyrasehemmer, Sulfonamide), Antidepressiva, Antikonvulsiva oder Antihistaminika haben delirogenes Potenzial.

\section{Diagnostik}

Zur raschen Diagnostik eignet sich die Confusion Assessment Method (CAM). Werden die Punkte 1, 2 sowie zusätzlich 3 oder 4 bejaht, liegt ein Delir vor:

1. Akuter Beginn, fluktuierender Verlauf

2. Störung der Aufmerksamkeit

3. Denkstörungen

4. Quantitative Bewusstseinsstörung. Ein Delir kann eine Abwärtsspirale in Gang setzen, die zum (weiteren) Autonomieverlust sowie zu bleibenden kognitiven Einschränkungen führen kann und mit hoher Komplikations- und Mortalitätsrate assoziiert ist.

\section{Nicht-medikamentöse Therapie}

Wichtig ist eine sichere und reizarme Umgebung. Sensorische Defizite (Brille, Hörgerät) müssen ausgeglichen, der Wechsel von Bezugspersonen und ungewöhnliche Körperwahrnehmungen (Blasenkatheter, Fixierung) vermieden werden. Günstig wirkt sich ein regelmäßiger Tagesablauf

Tab. 1 Abgrenzung zwischen Delir und Demenz

\begin{tabular}{|l|l|l|}
\hline \multicolumn{1}{|l|}{} & Delir & Demenz \\
\hline Psychiatrische Anamnese & meistens unauffällig & oft unauffällig \\
\hline Beginn & Stunden/Tage & Jahre \\
\hline Verlauf & fluktuierend & kontinuierlich \\
\hline Bewusstsein & getrübt & klar \\
\hline Aufmerksamkeit & reduziert & normal bis reduziert \\
\hline Orientierung & gestört & gestört \\
\hline Halluzination & häufig optisch & selten \\
\hline Wahn & häufig & gelegentlich \\
\hline Psychomotorik & verändert & meist normal \\
\hline Körperliche Symptome & meistens vorhanden & selten \\
\hline EEG & verändert & verändert \\
\hline
\end{tabular}

Haben Sie auch eine Frage an das consilium Geriatrie?

Haben Sie auch eine Frage, speziell aus den Bereichen Kognitive Störungen, Neurologie, Infektiologie, Schlafstörungen oder Dysphagie im Alter? Prof. Heppner und sein Expertenteam beantworten diese gerne. Das "consilium Geriatrie" ist ein gemeinsamer Service der MMW und des Unternehmens InfectoPharm.

Schicken Sie Ihre Frage kostenfrei an: geriatrie@consilium.services

aus. Diskussionen mit dem deliranten $\mathrm{Pa}$ tienten sind kontraproduktiv. Oft haben Angehörige einen günstigen Einfluss und können Vertrauen schaffen.

\section{Medikamentöse Therapie}

Bei Vorherrschen von Halluzinationen, Wahn und Denkstörungen kann Haloperidol oder Risperidon kurzfristig eingesetzt werden. Stehen psychomotorische Unruhe oder Schlafstörungen im Vordergrund, sind Pipamperon, Melperon oder Lorazepam in niedriger Dosis zu empfehlen. Bei Parkinson-Symptomatik oder Lewy-Body-Demenz ist Clozapin möglich (engmaschige Kontrollen von Blutbild und EKG!). Alternativ kann Quetiapin eingesetzt werden. Bei Patienten mit Epilepsie kann Melperon verabreicht werden, weil es die Krampfschwelle nicht erniedrigt.

\footnotetext{
$\rightarrow$ Literatur beim Verfasser

$\rightarrow$ Anschrift des Verfassers:

Univ.-Prof. Dr. med. H. J. Heppner, MHBA Klinik für Geriatrie, HELIOS Klinikum Schwelm, Witten/Herdecke, Dr.-MoellerStr. 15, D-58332 Schwelm E-Mail: hans.heppner@uni-wh.de
} 